\title{
Sofosbuvir/Velpatasvir Plus Ribavirin Combination Therapy for Patients with Hepatitis C Virus Genotype 1a, 2a, or 3b after Glecaprevir/Pibrentasvir Therapy Failed
}

\author{
Ayami Nonomura, Akihiro Tamori, Hoang Hai, Ritsuzo Kozuka, Hideki Fujii, \\ Sawako Uchida-Kobayashi, Masaru Enomoto and Norifumi Kawada
}

\begin{abstract}
:
Glecaprevir/pibrentasvir (GLE/PIB) is a pan-genotype anti-hepatitis C virus (HCV) therapy with high efficacy and safety. However, evidence supporting retreatment following failure of the GLE/PIB regimen is limited. We herein report 3 non-cirrhotic cases involving two men aged 51 and 58 years old and a woman aged 68 years old infected with $\mathrm{HCV}$ genotype 1a, 2a, and $3 \mathrm{~b}$ respectively who failed anti-HCV therapies including GLE/PIB therapy. With combination therapy of sofosbuvir/velpatasvir plus ribavirin (SOF/VEL+RBV) for 24 weeks, all 3 patients had achieved a sustained viral response (SVR) at 24 weeks after completing treatment. SOF/VEL+RBV therapy was effective for retreatment of HCV after failure of GLE/PIB therapy.
\end{abstract}

Key words: sofosbuvir/velpatasvir plus ribavirin, glecaprevir/pibrentasvir, hepatitis C virus, retreatment

(Intern Med 60: 3441-3445, 2021)

(DOI: 10.2169/internalmedicine.7028-21)

\section{Introduction}

Treatment for chronic hepatitis $\mathrm{C}$ virus (HCV) infection has progressed considerably since the development of directacting antivirals (DAAs) (1). In Japan, the combination of glecaprevir (GLE), an NS3/4A protease inhibitor, and pibrentasvir (PIB), an NS5A inhibitor, was approved as a pangenotypic DAA regimen in 2017. Several reports in realworld settings have demonstrated high sustained virological response (SVR) rates with GLE/PIB therapy among DAAnaïve patients infected with $\mathrm{HCV}$ genotype 1 and $2(2,3)$. GLE/PIB therapy can be effective for patients infected with $\mathrm{HCV}$ showing virologic failure after prior DAA therapies because of the antiviral activities of GLE and PIB against $\mathrm{HCV}$ strains carrying non-structural (NS) 3-resistanceassociated substitutions (RASs) or NS5A-RASs, except for the strains with NS5A-P32 deletion (4). The 2020 Japan Society for Hepatology guidelines for Management of Hepatitis C Virus Infection recommend 12-week GLE/PIB therapy for retreatment of patients who have failed prior treatment with protease and NS5A inhibitors (5). In several reports, due to the high anti-viral effect for HCV with RASs, GLE/ PIB therapy was shown to be effective for retreatment of patients infected with $\mathrm{HCV}$ genotype 1 who failed previous therapy with NS3 protease and NS5A inhibitors and patients with genotype 2 who failed previous treatment with sofosbuvir (SOF), an NS5B inhibitor and ribavirin (RBV) therapeutic $(6,7)$.

However, GLE/PIB therapy has reduced efficacy on HCV variants carrying NS5A-P32 deletion that emerge after DAA treatment failure $(4,6)$. The American Association for the Study of Liver Diseases (AASLD) and European Association for the Study of the Liver (EASL) recommend that patients who fail a DAA regimen containing an NS5A inhibitor be treated with fixed-dose combination therapy of SOF, the NS5B inhibitor velpatasvir (VEL) and the NS3/4A protease inhibitor voxilaprevir for 12 weeks $(8,9)$. One issue in Japan is that voxilaprevir is unavailable in clinical settings, and evidence supporting alternatives for the retreatment of patients who fail prior GLE/PIB therapy is limited.

$\mathrm{HCV}$ genotype $1 \mathrm{~b}$ accounts for approximately $70 \%$ of $\mathrm{HCV}$ infections in Japan, followed by genotypes $2 \mathrm{a}$ and $2 \mathrm{~b}$ at $20 \%$ and $10 \%$, respectively (10). Combination therapy of 
Table. Clinical Characteristics of the Patients at Baseline.

\begin{tabular}{lccc}
\hline & Case 1 & Case 2 & Case 3 \\
\hline Age (years) & 58 & 51 & 68 \\
Gender & Male & Male & Female \\
IL28B genotype (rs8099917 SNP) & TT & TT & TG \\
Cirrhosis/non-cirrhosis & Non-cirrhosis & Non-cirrhosis & Non-cirrhosis \\
History of HCC & None & None & None \\
IFN regimens & None & None & Peg-IFN+TVR+RBV \\
DAA regimens & SOF+RBV, GLE/PIB & GLE/PIB & GLE/PIB \\
Outcome after the latest DAA therapy & Relapse & Relapse & Breakthrough \\
HCV RNA viral load $(\log$ IU/mL) & 6.1 & 6.8 & 6.4 \\
HCV genotype & $1 \mathrm{a}$ & $2 \mathrm{a}$ & $3 \mathrm{~b}$ \\
RAS in NS5A & ND & L31M, P58S & Y93H \\
Leukocyte count $(/ \mu \mathrm{L})$ & 8,100 & 6,100 & 4,100 \\
Hemoglobin $(\mathrm{g} / \mathrm{dL})$ & 14.0 & 15.0 & 12.5 \\
Platelet count $\left(\times 10^{4} / \mu \mathrm{L}\right)$ & 25.0 & 26.2 & 13.1 \\
Total bilirubin $(\mathrm{mg} / \mathrm{dL})$ & 0.5 & 0.9 & 0.5 \\
ALT $(\mathrm{IU} / \mathrm{L})$ & 125 & 20 & 81 \\
FIB-4 index & 1.45 & 0.74 & 5.19 \\
AFP $($ ng/mL) & 2.9 & 2.5 & 15.3 \\
\hline
\end{tabular}

ND: not detected, IL28B: Interleukin 28B, SNP: single nucleotide polymorphism, HCC: hepatocellular carcinoma, IFN: interferon, DAA: direct-acting antivirals, ALT: alanine aminotransferase, AFP: alpha fetoprotein, GLE: glecaprevir, PIB: pibrentasvir, RAS: resistance-associated substitution, SOF: sofosbuvir, VEL: velpatasvir, RBV: ribavirin. TVR: telaprevir

$\mathrm{SOF} / \mathrm{VEL}+\mathrm{RBV}$ is a DAA regimen approved in 2019 for retreatment after the failure of prior DAA therapy. According to a recent report, chronic hepatitis patients who failed treatment with NS3 protease inhibitors and NS5A inhibitors, including GLE/PIB therapy, who had HCV genotype 1b carrying NS5A-P32 deletion variants achieved an SVR by combination therapy of SOF/VEL once daily plus weight-based RBV twice daily for 24 weeks (11). However, that report included only patients infected with HCV genotype $1 \mathrm{~b}$, which is dominant in Japan, so the implications concerning retreatment of patients with less common $\mathrm{HCV}$ genotypes who had failed prior GLE/PIB therapy were unclear.

We herein report three patients infected with HCV genotypes 1a, 2a, and $3 \mathrm{~b}$ who were successfully retreated with $\mathrm{SOF} / \mathrm{VEL}+\mathrm{RBV}$ therapy after failure of prior DAA therapies including GLE/PIB.

\section{Case Reports}

\section{Case 1}

The first case was a 58-year-old, non-cirrhotic man infected with $\mathrm{HCV}$ genotype $1 \mathrm{a}$ of $6.1 \mathrm{log} \mathrm{IU} / \mathrm{mL}$ at baseline (Table). He had received SOF/RBV therapy for HCV serotype 2 for 12 weeks and had once achieved an SVR at 24 weeks after the completion of treatment (SVR24). However, $\mathrm{HCV}$ relapsed 16 months after finishing SOF/RBV therapy and HCV genotype 1a was detected. Since the patient had been using illegal intravenous drugs, reinfection of HCV by drug injection was suspected. After stopping the illegal drug use, he was treated with GLE/PIB therapy for eight weeks.
Although the patient achieved SVR12, the level of HCV RNA was re-elevated at 24 weeks after finishing GLE/PIB therapy. Consequently, he visited our hospital to be treated for HCV again. A population-sequence analysis showed no RASs in the NS5A region of HCV genotype 1a after GLE/ PIB failure. The patient was treated with SOF/VEL once daily and $800 \mathrm{mg}$ RBV twice daily for 24 weeks. Despite hyperuricemia and mild liver transaminase elevation during the course, no serious adverse events were observed. At the 48th week after the end of SOF/VEL+RBV therapy, HCV RNA was undetectable.

\section{Case 2}

The second case was a 51-year-old, non-cirrhotic man infected with $\mathrm{HCV}$ genotype $2 \mathrm{a}$ at baseline level of $6.8 \mathrm{log}$ $\mathrm{IU} / \mathrm{mL}$ (Table). The patient was treated with GLE/PIB therapy for 8 weeks, after which his HCV RNA decreased to an undetectable level. However, HCV relapsed 11 weeks after finishing GLE/PIB therapy. Through a populationsequencing analysis, L31M and P58S in the NS5A region were detected before GLE/PIB therapy, and additional RASs in the NS5A region were not detected after the failure of GLE/PIB therapy. Subsequently, the patient was treated once daily with SOF/VEL and twice daily with $800 \mathrm{mg} \mathrm{RBV}$ for 24 weeks. Although the patient had lost $5 \mathrm{~kg}$ of weight during the therapy, he completed it. He also achieved an SVR 24.

\section{Case 3}

The third case was a 68-year-old, non-cirrhotic woman who was infected with $\mathrm{HCV}$ genotype $3 \mathrm{~b}$ at baseline level 
Case 1

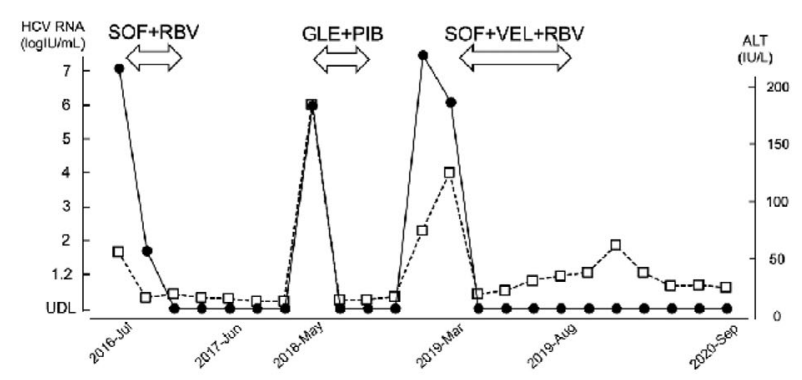

- hCVRna $\quad$ - alt

\section{Case 2}

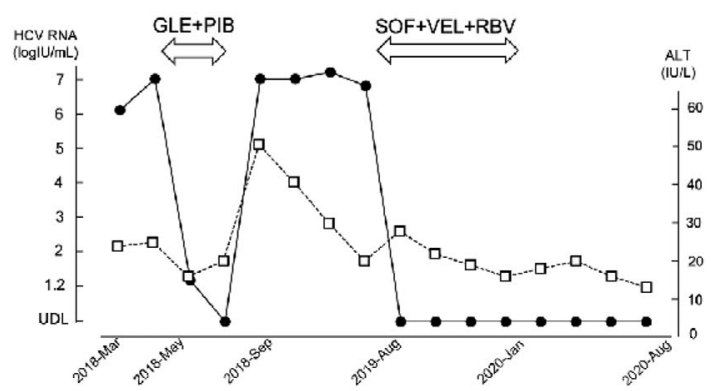

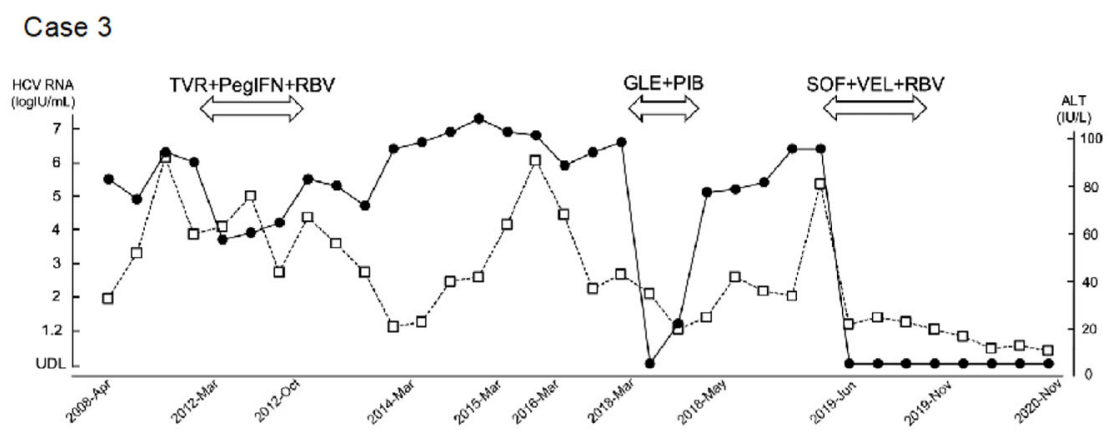

Figure. Clinical courses of anti-HCV therapies in three cases illustrated by changes in serum HCV RNA (solid line) and ALT (dotted line) levels. The lower limit of detection of HCV RNA was $1.2 \log$ IU/mL. ALT: alanine aminotransferase, GLE: glecaprevir, HCV: hepatitis C virus, PIB: pibrentasvir, RNA: ribonucleic acid, SOF: sofosbuvir, VEL: velpatasvir, RBV: ribavirin

of $6.4 \log \mathrm{IU} / \mathrm{mL}$ (Table). She was born in Japan and had never stayed abroad nor lived with a foreigner. She never had blood transfusions nor engaged in illicit drug injection either. She had a history of treatment with HCV serotype 1 with interferon (IFN)- $\alpha$ (human lymphoblastoid interferon), pegylated IFN+RBV, and telaprevir (TVR)+pegylated IFN+ $\mathrm{RBV}$, but these IFN-based therapies did not eliminate HCV.

After the failure of TVR+pegylated IFN+RBV, the genotype was examined. She was found to be infected with $\mathrm{HCV}$ genotype $3 b$, which did not match the serotype. Before GLE/PIB therapy, the histological findings had shown stage 2 and grade 2, non-cirrhosis. The patient was started on 12week GLE/PIB therapy; however, viral breakthrough occurred in the fourth week. After the failure of GLE/PIB, the ALT levels fluctuated around $40 \mathrm{U} / \mathrm{L}$ (Figure). At the start of SOF/VEL+RBV therapy, the alanine aminotransferase level was transiently elevated. The FIB-4 index was beyond 3.25 only at baseline. These data indicated advanced fibrosis that had not yet progressed to cirrhosis.

Population sequencing of the amino acid sequence of NS5 A of HCV genotype 3b showed S24-, K26-, M28-, K30-, M 31-, P32-, P58-, E92-, and Y93- before GLE/PIB therapy. After the failure of GLE/PIB therapy, Y93H replacement in NS5A was detected. The patient was treated with SOF/VEL plus $600 \mathrm{mg}$ of RBV for 24 weeks, and she achieved SVR 24. Mild pruritus and headache appeared during retreatment, but no serious adverse events were observed.

Notably, the serum HCV RNA levels dropped below the detectable limit on real-time polymerase chain reaction (PCR) by week 4 of the SOF/VEL+RBV therapy in all three of the above cases, and all of them achieved SVR24 (Figure).

\section{Discussion}

In a phase III trial in Japan, SOF/VEL+RBV therapy showed SVR12 rates of $98 \%$ for patients with genotype 1 and $92 \%$ for those with genotype 2 (12). However, only one patient in whom prior GLE/PIB therapy had failed was enrolled into that study. Recently, Takaki et al. reported that two of three patients with HCV genotype 1b carrying NS5 A-P32 deletion variants in whom prior GLE/PIB therapy had failed achieved an SVR after receiving SOF/VEL+RBV 24 weeks (11). In our report, three non-cirrhotic patients who were infected with HCV genotypes $1 \mathrm{a}, 2 \mathrm{a}$, and $3 \mathrm{~b}$ achieved SVR24 with SOF/VEL+RBV therapy after anti$\mathrm{HCV}$ therapies including GLE/PIB had failed.

In the real-world setting, GLE/PIB therapy has achieved high SVR rates in DAA-naïve patients with $\mathrm{HCV}$ genotypes 1 or 2 . According to recent studies, the total SVR rate was $99.5 \%(220 / 222,132 / 132$, and 830/834) in non-cirrhotic patients and $100 \%(48 / 48,41 / 41$, and 334/334) in cirrhotic patients $(7,13,14)$. A systematic review of GLE/PIB failure recently showed that baseline RASs in NS3 and NS5A were present in $44(88 \%)$ out of 50 cases of virologic failure (15). The presence of the baseline RASs L31M, P32del, and Y93 
$\mathrm{H}$ in NS5A reduced the odds of achieving SVR in patients with genotype 1 . In contrast, no significant RASs were detected in patients with genotype 2 .

In case 1 of our study, no RASs in the NS5A region of $\mathrm{HCV}$ genotype 1a were detected after GLE/PIB failure, whereas in case 2, which was infected with $\mathrm{HCV}$ genotype 2a, L31M and P58S in the NS5A region were detected at the baseline before GLE/PIB therapy. Case 1 was re-infected with HCV genotype 1a after SVR24 following treatment for HCV serotype 2, while case 2 did not have a history of DAA therapy. Therefore, these non-cirrhotic patients were treated with GLE/PIB therapy for eight weeks. However, real-world studies in Japan have shown that eight-week treatment was a common factor for GLE/PIB failure in DAA-naïve patients $(3,7,14)$. As we did not a perform sequence analysis of the NS3/4 protease region and deep sequencing in two cases, why initial DAA treatments failed in two cases is unclear; however, eight weeks of GLE/PIB therapy may have been insufficient for the therapy to be successful.

Case 3 was infected with genotype $3 b$, which is uncommon in Japan. The patient's source of infection could not be identified. She showed resistance to multiple anti-HCV therapies, including IFN-based regimens, and Y93H RAS emerged when viral breakthrough occurred during GLE/PIB therapy. In two clinical trials of GLE/PIB (VOYAGE-1 and VOYAGE-2), SVR12 was achieved in 95\% (19/20) of patients with genotype $3 \mathrm{a}$ and in $50 \%(10 / 20)$ of those with genotype $3 \mathrm{~b}$ (16). In Japanese studies, SVR12 was achieved in $100 \%(15 / 15)$ of patients with genotype $3 \mathrm{a}$ and in $40 \%$ $(4 / 10)$ of those with genotype $3 b(7,13,14)$. GLE/PIB therapy was shown to be less effective in patients with genotype $3 \mathrm{~b}$ than in those with genotype 3a. Generally, wild-type $\mathrm{HCV}$ genotype $3 \mathrm{~b}$ has a unique amino acid sequence in NS5 A: S24, M28, K30, M31, P32, P58, E92, and Y93 (17). This amino acid sequence might be one of the reasons for the reduced efficacy of GLE/PIB therapy in patients with genotype $3 b$.

In Case 3, SVR24 with SOF/VEL+RBV therapy was achieved, even though Y93H RAS emerged after the failure of GLE/PIB therapy. A clinical study on genotype 3 showed that $50 \%(2 / 4)$ of patients with $\mathrm{Y} 93 \mathrm{H}$ at baseline achieved SVR12 with SOF/VEL therapy. In contrast, of the 9 cirrhotic patients with $\mathrm{Y} 93 \mathrm{H}$ at baseline treated with SOF/VEL +RBV therapy, 8 (89\%) achieved SVR12 (18). In addition, Gane et al. reported that the rate of retreatment with $\mathrm{SOF} /$ VEL+RBV for 24 weeks was 78\% (14/18) in patients infected with genotype 3 in whom previous SOF/VEL-based regimens administered for 12 weeks had been ineffective (19). The addition of RBV and prolonged treatment duration were speculated to have led to SVR despite the previous failure of several therapies, including GLE/PIB.

Overall, SOF/VEL+RBV therapy was well tolerated in all cases. Although adverse events, such as hyperuricemia and mild liver transaminase elevation, weight loss, mild pruritus, and headache, were observed, no patient discontinued the therapy. A previous study reported that $74.8 \%$ of patients with HCV genotype 3 who were treated with SOF/VEL+ RBV for 12 weeks had adverse events, such as asthenia, headache, and insomnia. However, most adverse events were mild, and serious events were rare at $2 \%$ (18). Although the duration of our regimen was longer than in that study, most of the adverse events were mild, which was consistent with these previous findings.

In the United States of America and European countries, combination therapy with SOF/VEL/voxilaprevir is recommended for patients who fail to respond to prior treatment with a regimen containing an NS5A inhibitor $(8,9)$. However, voxilaprevir is not available in Japan. To our knowledge, there are no reports comparing the anti-HCV effects of SOF/VEL/voxilaprevir and SOF/VEL+RBV. Further studies are thus necessary to evaluate the effects of SOF/VEL+ $\mathrm{RBV}$ in patients with DAA failure.

In conclusion, we administered 24 weeks of SOF/VEL+ RBV therapy to 3 patients infected with genotypes other than $1 \mathrm{~b}$ in whom GLE/PIB therapy had failed. The therapy was safe and well-tolerated. SOF/VEL+RBV therapy was effective for patients who did not respond to multiple anti$\mathrm{HCV}$ therapy, regardless of HCV genotype.

All procedures followed have been performed in accordance with the ethical standards laid down in the 1964 Declaration of Helsinki and its later amendments. Informed consent for inclusion in this study was obtained from all patients.

Author's disclosure of potential Conflicts of Interest (COI). Norifumi Kawada: Research funding, AbbVie.

\section{References}

1. Spearman CW, Dusheiko GM, Hellard M, Sonderup M. Hepatitis C. Lancet 394: 1451-1466, 2019.

2. Ikeda $\mathrm{H}$, Watanabe T, Atsukawa M, et al. Evaluation of 8-week glecaprevir/pibrentasvir treatment in direct-acting antiviral-naïve noncirrhotic HCV genotype 1 and 2 infected patients in a realworld setting in Japan. J Viral Hepat 26: 1266-1275, 2019.

3. Ogawa E, Furusyo N, Nakamuta M, et al. Glecaprevir and pibrentasvir for Japanese patients with chronic hepatitis C genotype 1 or 2 infection: Results from a multicenter, real-world cohort study. Hepatol Res 49: 617-626, 2019.

4. Uemura H, Uchida Y, Kouyama JI, et al. NS5A-P32 deletion as a factor involved in virologic failure in patients receiving glecaprevir and pibrentasvir. J Gastroenterol 54: 459-470, 2019.

5. Drafting Committee for Hepatitis Management Guidelines, the Japan Society of Hepatology. Japan Society of Hepatology guidelines for the management of hepatitis C virus infection: 2019 update. Hepatol Res 50: 791-816, 2020.

6. Osawa M, Imamura M, Teraoka Y, et al. Real-world efficacy of glecaprevir plus pibrentasvir for chronic hepatitis C patient with previous direct-acting antiviral therapy failures. J Gastroenterol 54: 291-296, 2019.

7. Tamori A, Inoue $\mathrm{K}$, Kagawa $\mathrm{T}$, Takaguchi $\mathrm{K}$, et al. Intention-totreat assessment of glecaprevir+pibrentasvir combination therapy for patients with chronic hepatitis $\mathrm{C}$ in the real world. Hepatol Res 49: 1365-1373, 2019.

8. AASLD-IDSA HCV Guidance Panel. Hepatitis C guidance 2018 
update: AASLD-IDSA recommendations for testing, managing, and treating hepatitis C Virus Infection. Clin Infect Dis 67: 14771492, 2018.

9. European Association for the Study of the Liver. EASL recommendations on treatment of hepatitis $\mathrm{C}$ : final update of the series. J Hepatol 73: 1170-1218, 2020.

10. Kamae I, Kumada H, Kobayashi M, et al. Epidemiology, treatment, and health economics of Hepatitis C in Japan (Review). Kanzo 55: 589-603, 2014 (in Japanese, Abstract in English).

11. Takaki S, Imamura M, Yamaguchi S, et al. Real-word efficacy of sofosbuvir, velpatasvir plus ribavirin therapy for chronic hepatitis patients who failed to prior DAA therapy with NS5A-P32 deletion mutated HCV infection. Clin J Gastroenterol 13: 1233-1238, 2020.

12. Izumi $N$, Takehara $T$, Chayama $K$, et al. Sofosbuvir-velpatasvir plus ribavirin in Japanese patients with genotype 1 or 2 hepatitis $\mathrm{C}$ who failed direct-acting antivirals. Hepatol Int 12: 356-367, 2018.

13. Sezaki H, Suzuki F, Hosaka T, et al. Initial- and re-treatment effectiveness of glecaprevir and pibrentasvir for Japanese patients with chronic hepatitis C virus-genotype 1/2/3 infections. J Gastroenterol 54: 916-927, 2019.

14. Nozaki A, Atsukawa M, Kondo C, et al. The effectiveness and safety of glecaprevir/pibrentasvir in chronic hepatitis C patients with refractory factors in the real world: a comprehensive analysis of a prospective multicenter study. Hepatol Int 14: 225-238, 2020.

15. Singh AD, Maitra S, Singh N, et al. Systematic review with meta- analysis: impact of baseline resistance-associated substitutions on the efficacy of glecaprevir/pibrentasvir among chronic hepatitis C patients. Aliment Pharmacol Ther 51: 490-504, 2020.

16. Wei L, Wang G, Alami NN, et al. Glecaprevir-pibrentasvir to treat chronic hepatitis $\mathrm{C}$ virus infection in Asia: two multicentre, phase 3 studies- a randomised, double-blind study (VOYAGE-1) and an open-label, single-arm study (VOYAGE-2). Lancet Gastroenterol Hepatol 5: 839-849, 2020.

17. Krishnan P, Pilot-Matias T, Schnell G, et al. Pooled resistance analysis in patients with hepatitis $\mathrm{C}$ virus genotype 1 to 6 infection treated with glecaprevir-pibrentasvir in phase 2 and 3 clinical trials. Antimicrob Agents Chemother 62: e01249-18, 2018.

18. Esteban R, Pineda JA, Calleja JL, et al. Efficacy of sofosbuvir and velpatasvir, with and without ribavirin, in patients with hepatitis $\mathrm{C}$ virus genotype 3 infection and cirrhosis. Gastroenterology 155: 1120-1127, 2018.

19. Gane EJ, Shiffman ML, Etzkorn K, et al. Sofosbuvir-velpatasvir with ribavirin for 24 weeks in hepatitis $\mathrm{C}$ virus patients previously treated with a direct-acting antiviral regimen. Hepatology 66: 1083-1089, 2017.

The Internal Medicine is an Open Access journal distributed under the Creative Commons Attribution-NonCommercial-NoDerivatives 4.0 International License. To view the details of this license, please visit (https://creativecommons.org/licenses/ by-nc-nd/4.0/).

(C) 2021 The Japanese Society of Internal Medicine Intern Med 60: 3441-3445, 2021 